\title{
Usability of intraoperative cine-portogram during liver transplantation in young pediatric patients with biliary atresia
}

\author{
Shin Hwang, Jung-Man Namgoong, Gi-Young Ko, Seak Hee Oh, Kyung Mo Kim
}

Department of Surgery, Asan Medical Center, University of Ulsan College of Medicine, Seoul, Korea

Background: Pediatric patients with biliary atresia (BA) often present liver cirrhosis-associated portal hypertension and portal vein hypoplasia. For successful liver transplantation (LT), maintenance of sufficient portal inflow is essential through stenosis-free portal vein reconstruction and effective ligation of collaterals. This study was intended to assess the clinical usability of intraoperative cine-portogram (IOCP) in young pediatric patients who underwent LT for BA.

Methods: Medical records of pediatric patients younger than 10 years who underwent primary LT for BA from 2018 to 2020 were reviewed.

Results: All 31 patients had undergone Kasai portoenterostomy soon after birth. Median ages at Kasai portoenterostomy and LT were 1 month and 11 months, respectively. Types of LT were living donor LT in 13, deceased split LT in 15, and deceased whole LT in three patients. Portal vein interposition using iliac vein homograft was performed in all 28 patients receiving partial liver graft. Side-to-side portal vein unification venoplasty was performed in all three patients of whole LT. All patients underwent ligation of collateral veins. IOCP was performed in six (19.4\%). Four showed no or faint residual venous collaterals. Collateral vein embolization and endovascular stenting were performed in each one patient. Portal vein insufficiency-free survival rate was $100 \%$ at 1 year and $93.8 \%$ at 3 years. All patients are currently alive to date with a median follow-up period of 23 months.

Conclusions: IOCP can be a useful method for identification and embolization of the residual portosystemic collateral veins in young pediatric patients who undergo LT for BA.

Corresponding author: Shin Hwang

E-mail: shwang@amc.seoul.kr

(c) The Korean Society for Transplantation

This is an Open Access article distributed under the terms of the Creative Commons Attribution Non-Commercial License (http://creativecommons.org/licenses/by-nc/4.0/) which permits unrestricted non-commercial use, distribution, and reproduction in any medium, provided the original work is properly cited. 